( 2018 , The Authors. Published by FASS Inc. and Elsevier Inc. on behalf of the American Dairy Science Association ${ }^{\circledR}$.

This is an open access article under the CC BY-NC-ND license (http://creativecommons.org/licenses/by-nc-nd/4.0/).

\title{
Therapeutic effects of kefir grain Lactobacillus-derived extracellular vesicles in mice with 2,4,6-trinitrobenzene sulfonic acid-induced inflammatory bowel disease
}

\author{
M. K. Seo, ${ }^{1}$ E. J. Park, ${ }^{1}$ S. Y. Ko, E. W. Choi, and S. Kim ${ }^{2,3}$ \\ Prostemics Research Institute, Seongdong-gu, Seoul 04778, South Korea
}

\begin{abstract}
Kefir is a fermented product from yeast and lactic acid bacteria, and has been associated with various health benefits including relieving inflammatory bowel disease. Recently, it has been shown that gram-positive bacteria produce extracellular vesicles (EV). The EV could be appearing as potentially important mediators of cell to cell interaction. In this study, we explored the role of kefir grain Lactobacillus-derived EV in modulating inflammation responses via alleviating the production of inflammatory cytokines in tumor necrosis factor- $\alpha$ (TNF- $\alpha)$-induced inflammation in Caco-2 cells and the 2,4,6-trinitrobenzene sulfonic acid-induced inflammatory bowel disease mouse model. Kefir-derived Lactobacillus EV were isolated by ultracentrifugation of the culture medium of 3 different kefir-derived strains (i.e., Lactobacillus kefir, Lactobacillus kefiranofaciens, and Lactobacillus kefirgranum). Nanoparticle tracking analysis showed that the size of isolated kefir-derived Lactobacillus EV was within 80 to $400 \mathrm{~nm}$, and kefirderived Lactobacillus EV uptake into recipient Caco-2 cells was confirmed by fluorescence labeling. Treatment of each kefir-derived Lactobacillus EV onto TNF- $\alpha-$ stimulated Caco-2 cells significantly reduced the level of both mRNA expression and secretion of IL-8, and Western blot analysis revealed that such an effect was related to inhibition of TNF- $\alpha$ signaling mediated by reducing the phosphorylation of p65, a subunit of NF-kB. Subsequent administration of kefir-derived Lactobacillus EV into inflammatory bowel disease-induced mice significantly alleviated the body weight loss and rectal bleeding, and enhanced stool consistency. Histological examination showed that kefir-derived Lactobacillus EV substantially reduced the infiltration of transmural leukocytes and loss of goblet cells within the colon, and
\end{abstract}

\footnotetext{
Received May 4, 2018.

Accepted June 17, 2018.

${ }^{1}$ These authors contributed equally to this work.

${ }^{2}$ Current address: Stem Cell Center, Asan Institute for Life Sciences, Asan Medical Center, Seoul 05505, South Korea.

${ }^{3}$ Corresponding author: skim@amc.seoul.kr
}

the serum level of myeloperoxidase was significantly lower in the EV-treated group than control group. Our study demonstrates that kefir-derived Lactobacillus EV can be potentially used for developing innovative strategies for alleviating inflammatory bowel disease.

Key words: kefir, extracellular vesicle, functional dairy food, irritable bowel syndrome

\section{INTRODUCTION}

Kefir is a fermented milk product that originated in the Balkans, Eastern Europe, and the Caucasus (Fontán et al., 2006; Serafini et al., 2014). Kefir grains have a complex composition of microbial species; the predominant populations are lactic acid bacteria, acetic bacteria, yeasts, and fungi (Zhou et al., 2009; Pogačić et al., 2013). Some of the microorganisms isolated and identified in kefir cultures were classified using the product's name, as in Lactobacillus kefiri, Lactobacillus kefiranofaciens, Lactobacillus kefirgranum, Lactobacillus parakefir, and Candida kefyr (Wyder et al., 1999; Kwon et al., 2003; Yang et al., 2007; Taş et al., 2012). Kefir has various functions on human health due to its antimicrobial (Anselmo et al., 2010), immunoregulatory (Hong et al., 2009), antiallergenic (Hong et al., 2010), antitumoral (Gao et al., 2013), anti-inflammatory (Diniz et al., 2003), antidiabetic (Kwon et al., 2006), and antimutagenic (Guzel-Seydim et al., 2011) activities, although the mechanism of such function is not clear.

Inflammatory bowel disease (IBD) includes 3 entities (Crohn's disease, ulcerative colitis, and indeterminate colitis) characterized by a multifactorial pathogenesis and a chronic course. Inflammatory bowel disease is manifested by an inappropriate mucosal immune system response against intestinal luminal antigens (Farrell and LaMont, 2002; Sartor, 2004). Recently, Senol et al. (2015) demonstrated that kefir reduces the clinical disease activity index and histological colitis scores in a dextran sulfate sodium-induced colitis model, possibly via reduction of myeloperoxidase (MPO), tumor necrosis factor- $\alpha$ (TNF- $\alpha)$, and inducible nitric oxide synthase levels. A relevant study also showed that Lactobacillus subspecies, L. salivarius 433118, and Bifido- 
bacterium infantis 35624 were found to attenuate colitis in IL-10 knockout mice (McCarthy et al., 2003).

It has been reported that the release of biological cargos including nucleic acids, toxins, and enzymes is mediated by extracellular vesicles (EV) in grampositive bacteria, suggesting the highly conserved cellular transport system in various species (Deatherage and Cookson, 2012; Brown et al., 2015). Functionally $\mathrm{EV}$ are reported to be involved in numerous biological processes including the elimination of unwanted proteins or molecules from the cell, exchange of molecular materials between cells, communication between cells, and antigen presentation (Deatherage and Cookson, 2012). Various types of gram-negative bacteria have been shown to produce outer membrane vesicles containing a polysaccharide, DNA, immunomodulatory compounds, and communication factors (Zhou et al., 1998; Schwechheimer et al., 2013). Although the EV were discovered in gram-negative bacteria decades ago (Mashburn-Warren and Whiteley, 2006; Lee et al., 2008), studies in gram-positive bacteria have been completely overlooked until recently. This can be attributed to the structural traits of gram-positive bacteria such as Staphylococcus aureus, Bacillus subtilis, and Lactobacillus, whose plasma membrane is surrounded by a much thicker cell wall. However, recent studies have shown that gram-positive bacteria produce biologically active EV (Rivera et al., 2010; Gurung et al., 2011).

The aim of this study was to examine whether EV derived from Lactobacillus kefir, Lactobacillus kefiranofaciens, or Lactobacillus kefirgranum can mediate the role of these functional bacteria in alleviating the production of inflammatory cytokine in TNF- $\alpha$-induced inflammation in Caco-2 cells, and to investigate the protective effects of kefir-derived Lactobacillus EV in 2,4,6-trinitrobenzenesulfonic acid (TNBS)-induced IBD in a mouse model. Our results will provide essential information on understanding the mechanism of EV-mediated role of functional bacterium in reducing inflammation.

\section{MATERIALS AND METHODS}

\section{Cell Culture and Treatment}

Caco-2 cells were cultured at $37^{\circ} \mathrm{C}$ under a $5 \% \mathrm{CO}_{2}$ humidified atmosphere in Eagle's minimum essential medium containing 100 units/mL of penicillin, $100 \mu \mathrm{g} /$ $\mathrm{mL}$ of streptomycin, and $10 \%$ fetal bovine serum. Commercially available TNF- $\alpha$ (Sigma-Aldrich, H8916) was dissolved in 100\% dimethyl sulfoxide to a final concentration of $2 \mu \mathrm{g} / \mathrm{mL}$ of TNF- $\alpha(20 \mathrm{ng} / \mathrm{mL})$, which was identified as the optimal dose for stimulation experiments. The culture medium containing the TNF- $\alpha$ was removed after $6 \mathrm{~h}$ of pretreatment, and a fresh culture medium containing kefir-derived Lactobacillus (L. kefir, L. kefiranofaciens, and L. kefirgranum) EV was added for $24 \mathrm{~h}$.

\section{Preparation of Kefir-Derived Lactobacillus EV}

Secreted EV were isolated from kefir culture medium by differential centrifugation essentially as previously described (Oliveira et al., 2010). Lactobacillus kefir KCTC 3611, L. kefiranofaciens KCTC 5075, and L. kefirgranum KCTC 5086 were obtained from the Korean Collection for Type Cultures (KCTC). All strains were maintained and cultured on MRS medium (Difco, Franklin Lakes, NJ) at pH 5.2 to 7.0 under anaerobic conditions unless otherwise mentioned. Conditioned medium was collected after $96 \mathrm{~h}$ and was centrifuged at $300 \times g$ at $4^{\circ} \mathrm{C}$ for $10 \mathrm{~min}$ to eliminate bacteria. The supernatant was centrifuged at $1,200 \times g$ at $4^{\circ} \mathrm{C}$ for 20 min and $10,000 \times g$ at $4^{\circ} \mathrm{C}$ for $30 \mathrm{~min}$. The $\mathrm{EV}$ pellet was collected by ultracentrifugation at $100,000 \times g$ at $4^{\circ} \mathrm{C}$ for $70 \mathrm{~min}$ and suspended in PBS. All samples were filtered through a $0.22-\mu \mathrm{m}$ syringe filter (Merck Millipore, Darmstadt, Germany).

\section{Characterization of Kefir-Derived Lactobacillus EV}

Real-time high-resolution particle detection, counting, and sizing were performed on the NanoSight NS300 following manufacturer protocols (Malvern Instruments, Malvern, UK). Particle concentration (particles/ $\mathrm{mL}$ ) of kefir-derived Lactobacillus EV was calculated by the NanoSight system. The Nanoparticle Tracking Analysis system was also used to compare changes in concentrations and sizes before and after ultracentrifugation of EV in kefir-derived Lactobacillus culture medium. For cryo-transmission electron microscopy images, grid (200 mesh R 2/2 Quantifoil holey-carbon) was vitrified using a Vitrobot Mark III (Leica, Wetzlar, Germany) at room temperature and $100 \%$ humidity. A $4-\mu \mathrm{L}$ droplet of the vesicle suspension was applied to the grid. Excess sample was removed by blotting once between 1 and $2 \mathrm{~s}$ with filter paper. The blotted grid was plunged into liquid ethane and transferred under liquid nitrogen. Sample analysis was carried out under an FEI transmission cryoelectron microscope (Tecnai F20 G2, FEI Corp., Hillsboro, OR) with an acceleration voltage of $200 \mathrm{kV}$. Images were recorded with a $2 \mathrm{k} \times 2 \mathrm{k}$ Gatan Ultrascan 1000 CCD camera (Gatan, Pleasanton, CA). Kefir-derived Lactobacillus EV were isolated as described above and labeled with PKH26 (Sigma-Aldrich, St. Louis, MO) for $10 \mathrm{~min}$ at room temperature. Labeled EV were washed twice with PBS and resuspended in complete medium. Caco-2 
cells were grown on coverslips and treated with $20 \mu \mathrm{g} /$ $\mathrm{mL}$ of PKH26-labeled EV for $3 \mathrm{~h}$ at $37^{\circ} \mathrm{C}$. Nuclei were stained with Hoechst 33342 (Molecular Probes/Life Technologies, Carlsbad, CA) and analyzed by confocal microscopy.

\section{Determination of IL-8 Expression in Caco-2 Cells}

Caco-2 cells in 24-well plates were incubated with the indicated concentrations of kefir-derived Lactobacillus $\mathrm{EV}$ for $24 \mathrm{~h}$ after being stimulated with TNF- $\alpha$ for $6 \mathrm{~h}$. The RNA was isolated using Trizol (Invitrogen, Karlsruhe, Germany). The RNA was reverse transcribed using the Maxime RT premix (Intron, Gyeonggi-do, Korea). The synthesized cDNA was amplified using SYBR Premix Ex TaqII (Takara Bio) and analyzed using the StepOnePlus Real-Time PCR system. The designed primers in this study were as follows: GAPDH forward primer, 5'-TGTGGGCATCAATGGATTTGG-3'; GAPDH reverse primer, 5'-ACACCATGTATTCCGGGTCAAT-3'; IL-8 forward primer, 5'-ACTGAGAGTGATTGAGAGTGGAC-3'; IL-8 reverse primer, 5'-AACCCTCTGCACCCAGTTTTC-3'. The cycling conditions were as follows: 1 cycle at $95^{\circ} \mathrm{C}$ for $3 \mathrm{~min}$ followed by 40 cycles each at $95^{\circ} \mathrm{C}$ for $20 \mathrm{~s}$ and $60^{\circ} \mathrm{C}$ for $10 \mathrm{~s}$. To normalize the amount of total RNA present in each reaction, the GAPDH gene was used as an internal standard.

\section{Determination of IL-8 Secretion by Caco-2 Cells}

To determine IL- 8 secretion, $1 \times 10^{9}$ particles $/ \mathrm{mL}$ of kefir-derived Lactobacillus EV were cultured with Caco- 2 cells for $24 \mathrm{~h}$, and the conditioned medium was collected. Conditioned medium aliquots were centrifuged to remove cellular debris and then IL- 8 protein concentrations were quantified using the ELISA kit (R\&D Systems, Minneapolis, MN), according to the manufacturer's protocol (https://www.rndsystems .com/products/human-il-8-cxcl8-quantikine-elisa-kit _d8000c\#assay-procedure). The ELISA 96-well microplates were analyzed using a microplate reader at 570 nm (Bio-Tek, Winooski, VT).

\section{Protein Determination by Western Blotting}

Caco-2 cells were treated for $24 \mathrm{~h}$ with an even mixture of L. kefir, L. kefiranofaciens, and L. kefirgranum $\mathrm{EV}\left(1 \times 10^{9}\right.$ particles $\left./ \mathrm{mL}\right)$. For positive control, cells cultured with EV in the presence of $5 \mu M$ budenoside (Sigma-Aldrich, St. Louis, MO) were used. To detect the NF- $\kappa \mathrm{B}$ protein, cells were collected and lysed in radio immunoprecipitation assay buffer $(50 \mu \mathrm{L})$. After centrifugation at $20,000 \times g$ for $20 \mathrm{~min}$ at $4^{\circ} \mathrm{C}$ for removal of cell debris, the pellet was discarded. Cell lysate in radio immunoprecipitation assay buffer was mixed with NuPAGE LDS sample buffer $(5 \times$; Invitrogen, Carlsbad, CA) and heated at $95^{\circ} \mathrm{C}$ for $10 \mathrm{~min}$. Equal amounts of protein $(40 \mu \mathrm{g})$ were separated by SDS-PAGE and transferred to polyvinylidene difluoride filters (Protein Sequencing Membrane). After blocking overnight at $4^{\circ} \mathrm{C}$ with $5 \%$ nonfat milk, the blots were probed with primary antibodies for overnight at $4^{\circ} \mathrm{C}$ with gentle shaking. Phospho-NF-кB p65 (Ser536; 93H1) and GAPDH (D16H11) were purchased from Cell Signaling (Danvers, MA). After incubation of the membrane with the appropriate peroxidase-conjugated secondary antibodies (Abcam) for $1 \mathrm{~h}$ at room temperature, the reactive protein signals were visualized and evaluated by using the enhanced chemiluminescence system (Thermo Fisher Scientific, Waltham, MA).

\section{Animals}

Male, 8-wk-old Balb/cAnNCrljOri mice were acquired from Orient Bio Inc. (Gyeonggi-do, Korea). Mice were housed in an animal care facility, and were provided with food and water ad libitum; they were exposed to a $12 \mathrm{~h}$ light $/ 12 \mathrm{~h}$ dark cycle at $23 \pm 3{ }^{\circ} \mathrm{C}$. All procedures were approved by The Animal Care and Use Committee of KNOTUS IACUC (the Institutional Animal Care and Use Committee at the KNOTUS Co. Ltd), Gyeonggi-do, Korea (approval no. KNOTUS IACUC 16-KE-154).

\section{Mouse Model of TNBS-Induced IBD}

The IBD was induced by the rectal administration of $0.1 \mathrm{~mL}$ of $2.5 \%$ TNBS (Sigma-Aldrich) in $50 \%$ ethanol using a $3.5 \mathrm{~F}$ catheter positioned $3.5 \mathrm{~cm}$ proximal to the anus. During the procedure, the mice were anesthetized using Zoletil 50 (Virbac, Carros, France, $5 \mathrm{mg} / \mathrm{kg}$ ) and xylazine (Rompun, Bayer AG, Leverkusen, Germany, $2.5 \mathrm{mg} / \mathrm{kg}$ ). Following the installation of the catheter, the animals were kept vertical for $1 \mathrm{~min}$. The normal control groups $(\mathrm{n}=5)$ were administered $50 \%$ ethanol instead TNBS rectally. An even mixture of L. kefir, $L$. kefiranofaciens, and L. kefirgranum EV $\left(3 \times 10^{8}\right.$ or 3 $\times 10^{10}$ particles/head, $\mathrm{n}=5$ in each group, $1 \mathrm{~mL}$ in saline), vehicle (saline water, $\mathrm{n}=5$ ), or positive control (prednisolone, $2 \mathrm{mg} / \mathrm{kg}, \mathrm{n}=5$ ) were orally administrated for $10 \mathrm{~d}$ and the mice were monitored daily for BW, rectal bleeding, and stool consistency. The evaluation criteria were as follows: (1) stool blood, 0: negative, 2: fecal occult blood test positive, 4: gross bleeding; (2) stool consistency, 0: negative, 2: loose stool, 4: diarrhea. As scheduled, the animals were euthanized by means of exsanguination from the abdominal vena cava. This 
procedure was performed under anesthesia using Zoletil 50 (Virbac, $5 \mathrm{mg} / \mathrm{kg}$ ) and xylazine (Rompun, Bayer AG, Germany, $2.5 \mathrm{mg} / \mathrm{kg}$ ), and all efforts were made to minimize suffering. All animal experiments were approved by the Animal Care and Use Committee of KNOTUS IACUC, Gyeonggi-do, Korea (approval no. KNOTUS IACUC 16-KE-154).

\section{Histological Analysis}

The colonic tissues were fixed in $4 \%$ paraformaldehyde and embedded in paraffin. Fixed tissues were cut into 5-mm-thick sections, placed on glass slides, and deparaffinized. The sections were stained with hematoxylin and eosin and observed under a light microscope (Olympus BX53, Tokyo, Japan). The evaluation criteria are as follows: no evidence of inflammation (score 0), low level of inflammation with scattered infiltrating mononuclear cells (1-2 foci, score 1$)$, moderate inflammation with multiple foci (score 2), high level of inflammation with increased vascular density and marked wall thickening (score 3), and maximal severity of inflammation with transmural leukocyte infiltration and loss of goblet cells (score 4).

\section{In Vivo Biodistribution of Kefir-Derived Lactobacillus EV}

The EV isolated from culture supernatant-derived kefir were incubated with $1 \mu \mathrm{L}$ of $10 \mathrm{mM}$ Flamma675 for $2 \mathrm{~h}$ in dark. The EV were washed with $10 \mathrm{~mL}$ of PBS and subjected to ultracentrifugation, and this process was repeated twice. An even mixture of L. kefir, L. kefiranofaciens, and L. kefirgranum $\mathrm{EV}$, at a concentration of $1 \times 10^{10}$ particles in $1 \mathrm{~mL}$ of PBS, was orally administered in mice. Migration of fluorescently labeled $\mathrm{EV}$ in mouse organs was detected using an IVIS systems (Perkin Elmer, Waltham, MA) after 24 h. During the procedure, the mice were anesthetized using Zoletil 50 (Virbac, $5 \mathrm{mg} / \mathrm{kg}$ ) and xylazine (Rompun, Bayer AG, Germany, $2.5 \mathrm{mg} / \mathrm{kg}$ ).

\section{Myeloperoxidase Activity Assays}

Plasma myeloperoxidase (MPO) activity was determined using commercially available ELISA kit Mouse MPO ELISA Kit (EIAab Science Co. Ltd., Wuhan, China) according to the manufacturer's instruction (http://www.eiaab.com/entries/steps/ELISA\%20Kit/ PERM_MOUSE/Mouse).

\section{Statistical Analyses}

Data are representative of 3 or more independent experiments. One-way ANOVA test followed by paired $t$-test was used for statistical analysis, and $P<0.01$ or 0.05 was considered significant.

\section{RESULTS}

\section{Characterization of Kefir-Derived Lactobacillus EV}

We isolated EV from the supernatant of L. kefir, L. kefiranofaciens, and L. kefirgranum culture medium. The isolated particles were characterized by nanoparticle tracking analysis (NTA), cryoelectron microscopy, and fluorescent labeling dye. The size distribution of EV ranged between 80 and $400 \mathrm{~nm}$ (Figure 1A), and cryo-transmission electron microscopy images exhibited a nano-sized vesicle with a lipid bilayer (Figure 1B). Also, the uptake of these EV into Caco-2 cells was confirmed (Figure 1C).

\section{Anti-Inflammatory Effect of Kefir-Derived Lactobacillus EV in Cells}

Next, we investigated whether kefir-derived Lactobacillus EV had anti-inflammatory activity in Caco-2 cells. As shown in Figure 2A, EV collected from each kefir grain reduced the expression of IL-8 in TNFtreated cells (Figure 2A). In addition, combinatory treatment with EV harvested from 3 kefirs reduced the expression of IL- 8 and TNF- $\alpha$ more drastically compared with being treated with EV from respective kefir species (Figure 2B). Similarly, the secretion of IL-8 from Caco-2 cells was also inhibited after being treated with kefir-derived Lactobacillus EV (Figure $2 \mathrm{C})$. Western blot analysis showed that the mixture of L. kefir, L. kefiranofaciens, and L. kefirgranum EV (A mix ratios: 1:1:1) inhibited the TNF- $\alpha$-mediated increase of phosphorylated p65 (Figure 2D), a subunit of NF-kB. Collectively, these data suggest that the 3 mixed EV inhibit the expression and production of IL-8 via blocking the phosphorylation of p65 (Figure 2D).

\section{Effect of Kefir-Derived Lactobacillus EV Mixture on Histological Damage in Colon Tissues of Mice with IBD}

As shown in Figure 3A, the loss of BW by TNBS injection was significantly inhibited after being treated with the mixture of L. kefir, L. kefiranofaciens, and $L$. kefirgranum EV (A mix ratios: 1:1:1). Similarly, the incidence of rectal bleeding was significantly reduced by 75.0 and $87.5 \%$ in animals treated with $3 \times 10^{8}$ and $3 \times 10^{10}$ the 3 mixed EV, respectively (Figure $3 \mathrm{~B}$ ). Lastly, stool consistency was improved in the animals treated with the 3 mixed EV compared with the vehicle control from $6 \mathrm{~d}$ of treatment (Figure 3C). 
A

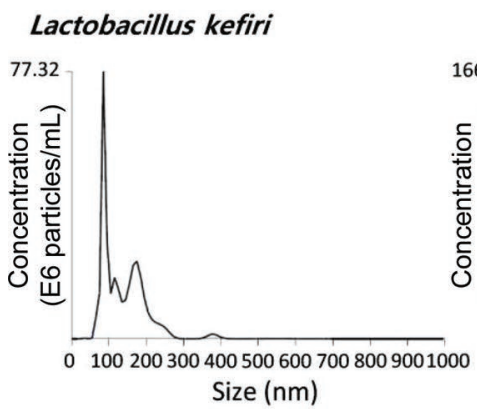

L. kefiranofaciens

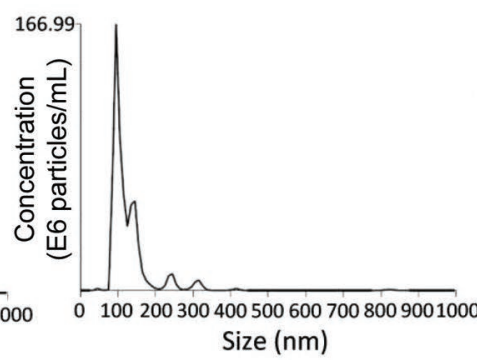

B

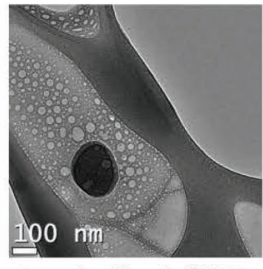

Lactobacillus kefiri EV

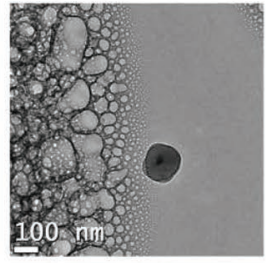

L. kefiranofaciens EV
L. kefirgranum EV

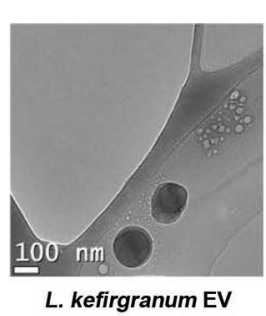

C Dye-only control

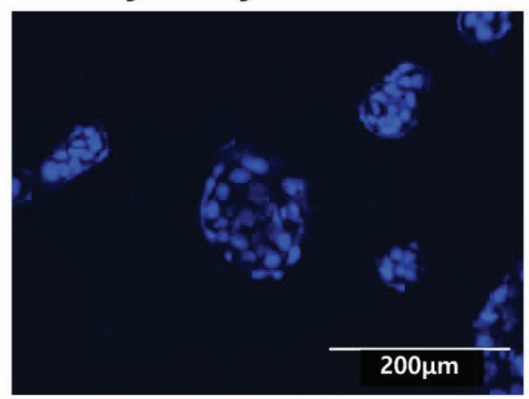

L. kefiranofaciens EV

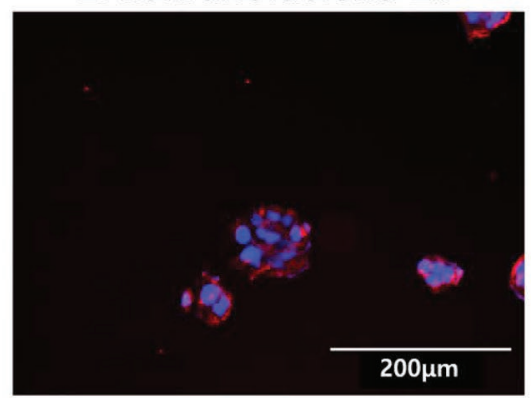

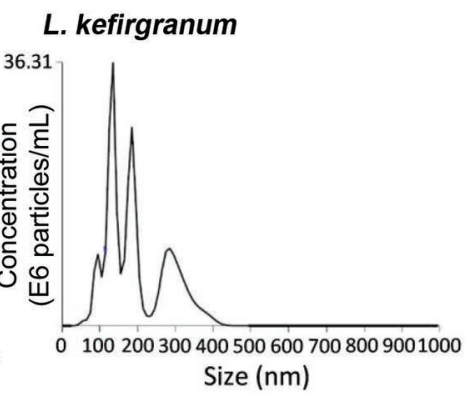

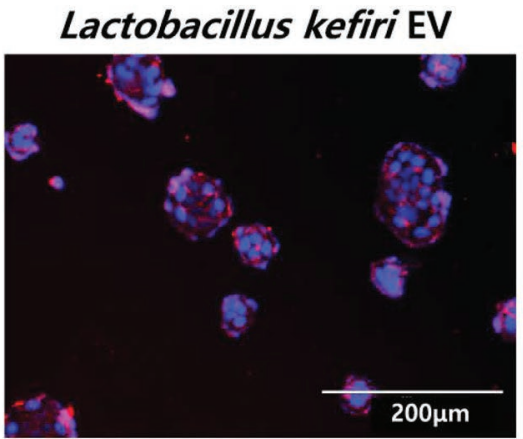

\section{L. kefirgranum EV}

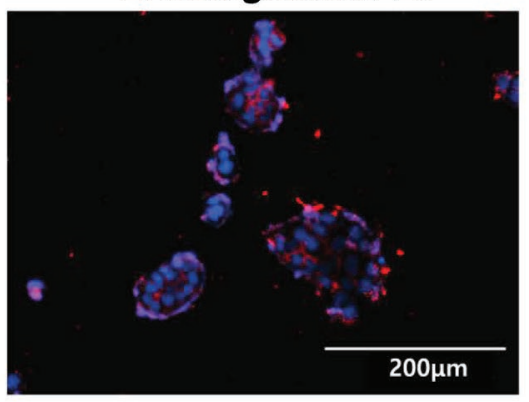

Figure 1. Kefir-derived Lactobacillus extracellular vesicle (EV) characterization. Particle size distribution and concentration of kefir-derived Lactobacillus EV. The EV were resuspended in $100 \mu \mathrm{L}$ of PBS and used for analyses (A). Cyro-transmission electron microscopy imaging of isolated kefir-derived Lactobacillus EV (B). The fluorescence image shows that kefir-derived Lactobacillus EV were uptaken into Caco-2 cells. Cultured Caco-2 cells were incubated with PKH26-labeled kefir-derived Lactobacillus EV for 3 h and the image was taken. Blue, cell nuclei; red, PKH26-labeled kefir-derived Lactobacillus EV. Numerous labeled EV were observed in the cells (C).

\section{Kefir-Derived Lactobacillus EV Mixture Reduced Inflammatory Response in Mice with IBD}

We next investigated whether kefir-derived Lactobacillus extracellular vesicle mixture has a therapeutic function in IBD colitis model. As shown in Figures 4A and $4 \mathrm{~B}$, the 3 mixed EV substantially inhibited TNBSinduced infiltration of transmural leukocyte and loss of goblet cells. We have shown the anti-inflammatory effect of the 3 mixed EV in mice with TNBS-induced colitis. To whether the 3 mixed EV reached the colon, the biodistribution and intestinal absorption of the 
A

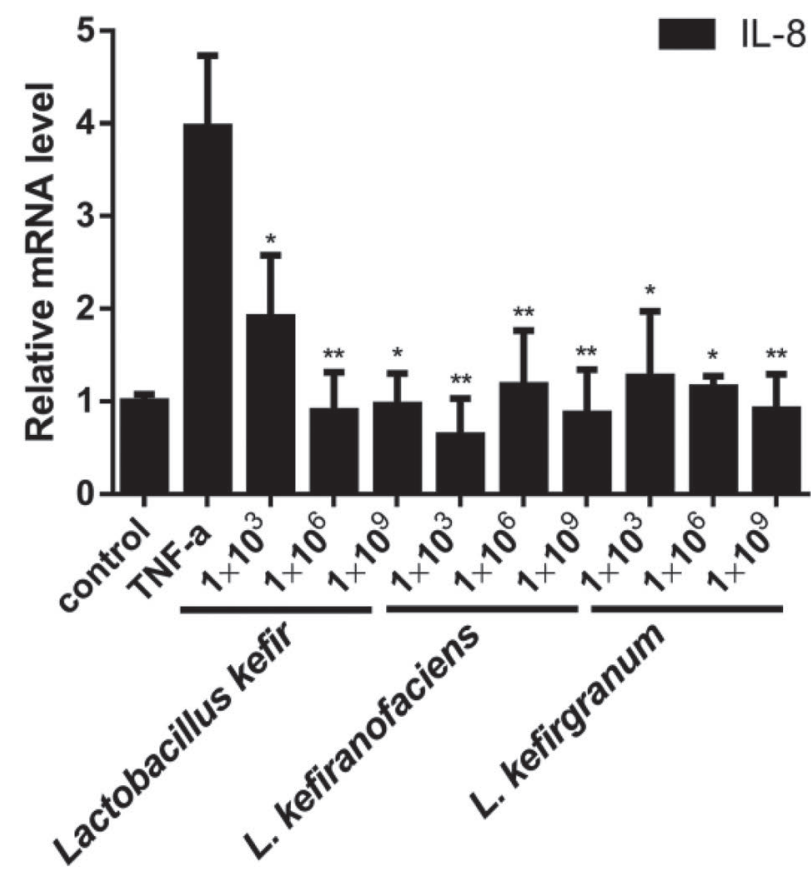

B

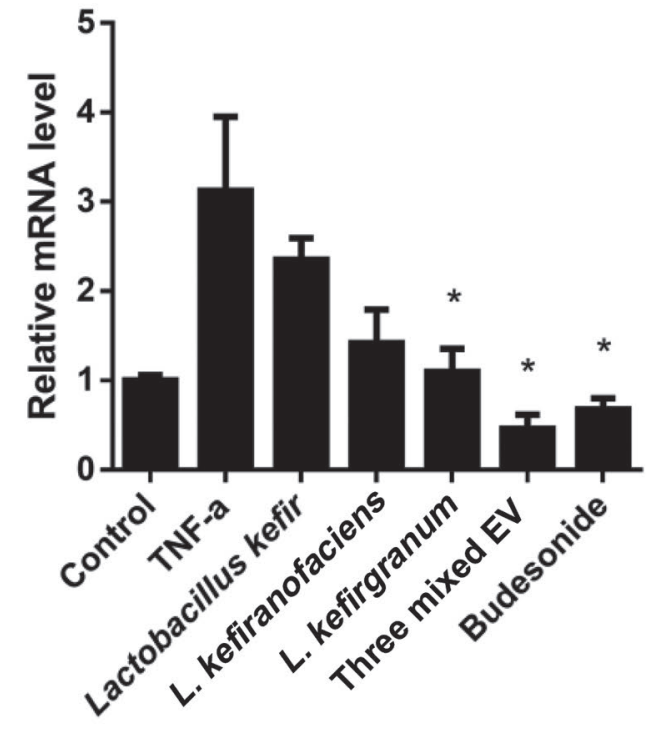

C

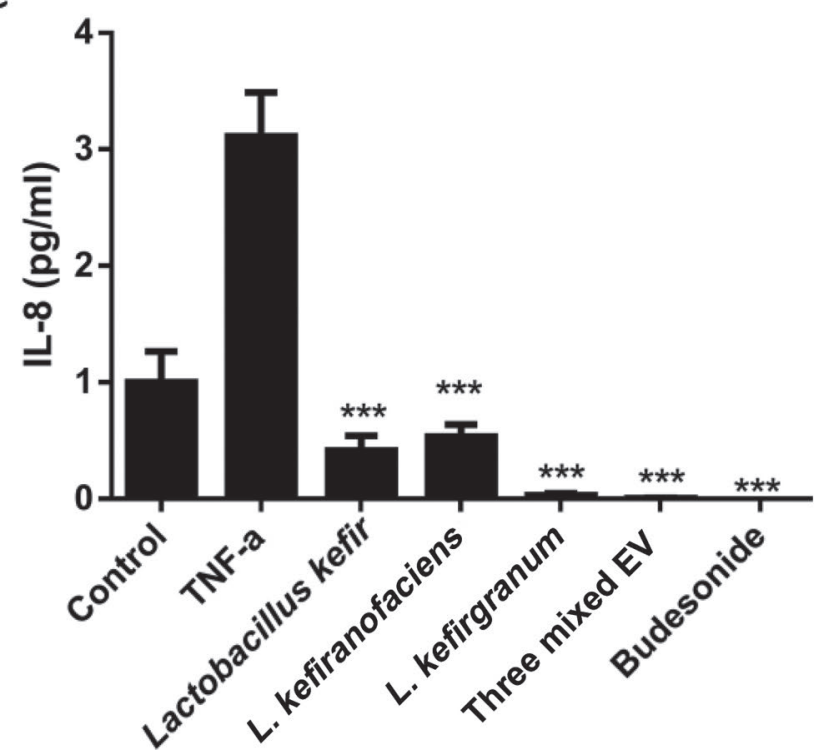

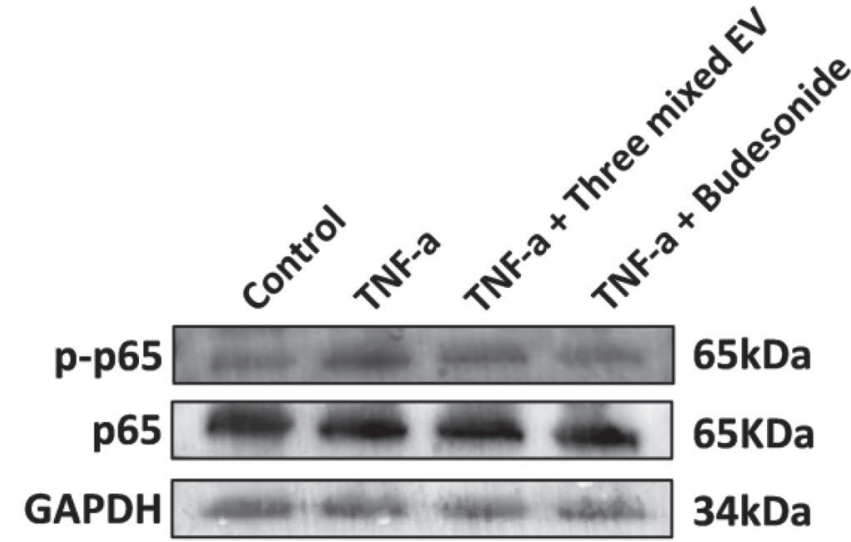

Figure 2. Effect of kefir-derived Lactobacillus extracellular vesicles (EV) on anti-inflammatory effects in cells. Effect of kefir-derived Lactobacillus EV on the mRNA level of IL-8 in tumor necrosis factor- $\alpha$ (TNF- $\alpha$ ) stimulated Caco-2 cells. The expression of IL-8 was decreased significantly by kefir-derived Lactobacillus EV (A). The synergistic effect of Lactobacillus kefir, Lactobacillus kefiranofaciens, and Lactobacillus kefirgranum was confirmed by IL-8 expression (B). Three mixed EV represent an even mixture of EV collected from 3 species of bacteria. Interleukin- 8 concentrations were decreased by being treated with kefir-derived Lactobacillus EV (C). Western blot analysis of p-p65 after being treated with the mixture of L. kefir, L. kefiranofaciens, and L. kefirgranum EV (A mix ratios: 1:1:1) in Caco-2 cells. For positive control, budenoside ( $5 \mu M$; Sigma-Aldrich, St. Louis, MO) was added in the culture medium (D). Data are presented as the means \pm SD ( $\mathrm{n}=3$ ). Asterisks indicate a significant difference at ${ }^{* * *} P<0.001,{ }^{* *} P<0.01,{ }^{*} P<0.05$ compared with the TNF- $\alpha$ stimulated Caco-2 cells. 
orally administered Flamma675-labeled the 3 mixed EV was investigated by the IVIS Spectrum In Vivo Imaging System. The signal was clearly visualized in the intestinal region of Flamma675-labeled EV rats for $24 \mathrm{~h}$ (Figure 4C). Finally, the increase of serum MPO in TNBS-induced IBD colitis mice was blocked by the 3 mixed EV treatment (Figure 5).

\section{DISCUSSION}

Typically, few studies have reported on collecting EV from gram-positive bacteria including kefir-derived lactobacillus compared with those from gram-negative bacteria (Kim et al., 2013) because investigators thought that the thick cell wall of gram-positive bacteria might inhibit the production of EV (Brown et al., 2015). However, a recent study has described that Staphylococcus aureus, which is a gram-positive bacterium, produces EV with a distinct proteomic profile. This recent study showed that the sizes of EV released from Staphylococcus aureus were 20 to $100 \mathrm{~nm}$ in diameter (Lee et al., 2009). Similarly, EV from other gram-positive bacteria including Bacillus spp., Clostridium perfringens, and Streptomyces coelicolor were identified, and their average size ranged from 20 to $400 \mathrm{~nm}$ in diameter (Lee et al., 2009; Brown et al., 2015). In the present study, we
A

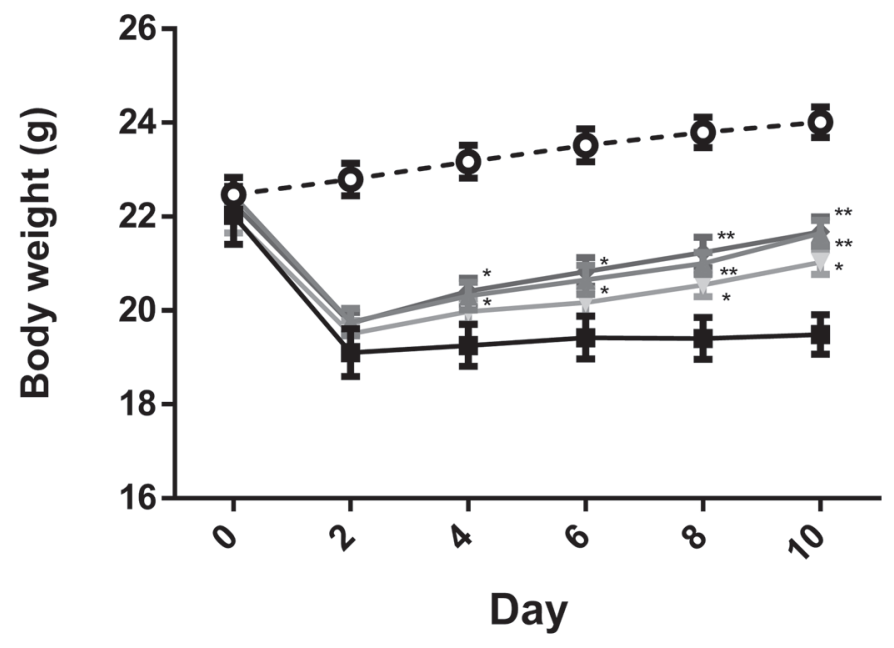

B

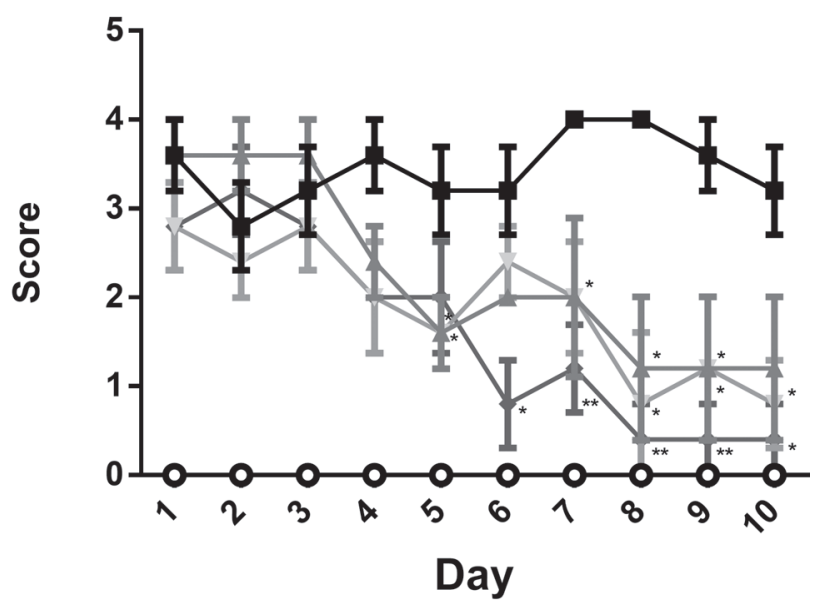

C Stool consistency

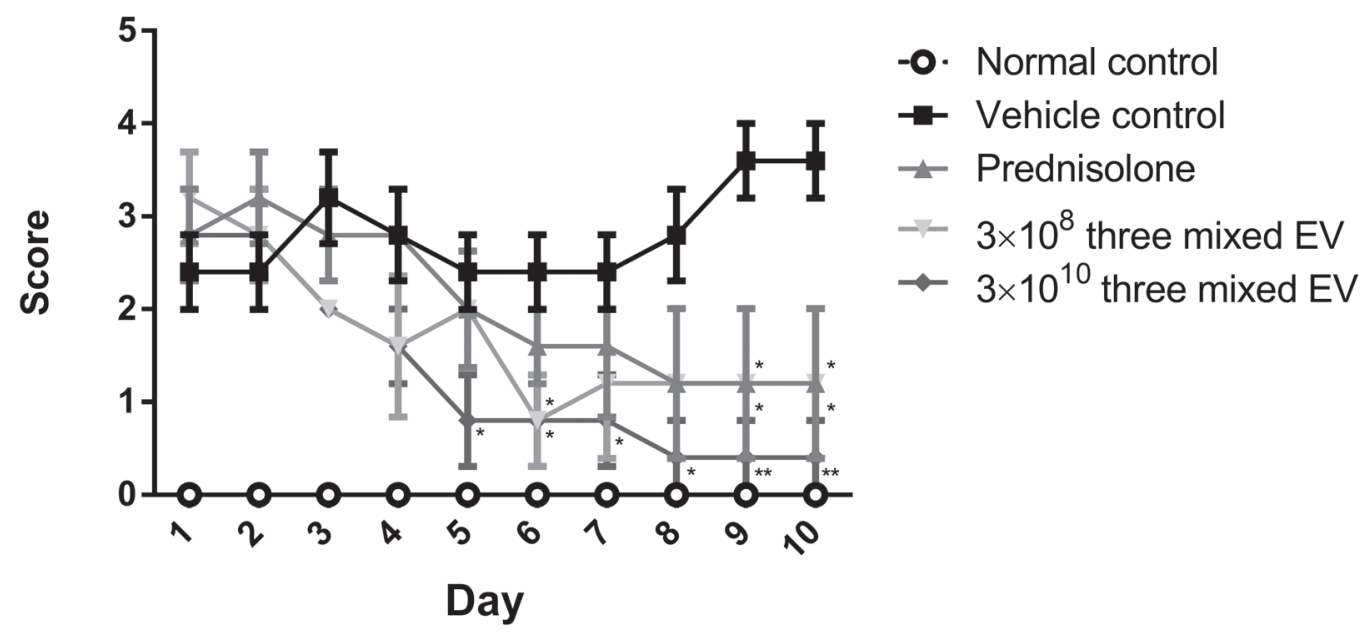

Figure 3. Therapeutic effects of kefir-derived extracellular vesicles (EV) on 2,4,6-trinitrobenzenesulfonic acid-induced mice with inflamma-

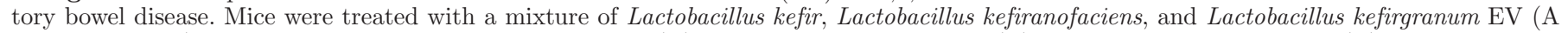

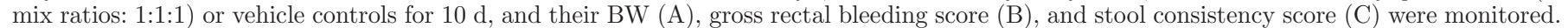

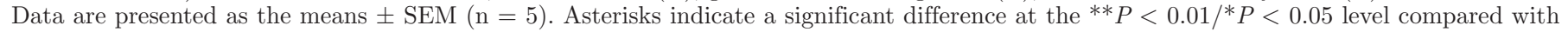
the vehicle control. 
A

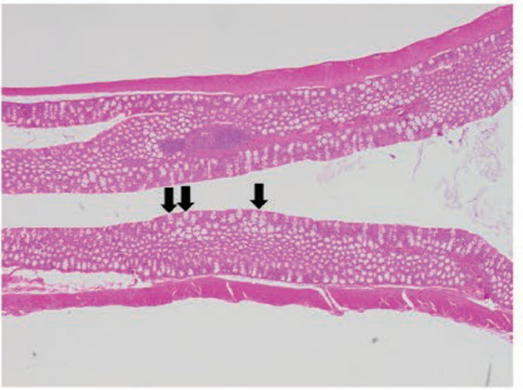

Normal control

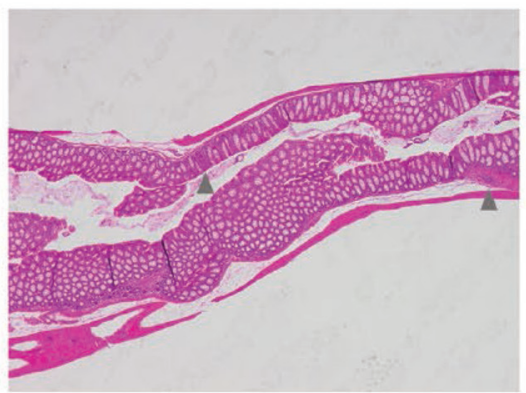

$3 \times 10^{8}$ three mixed EV

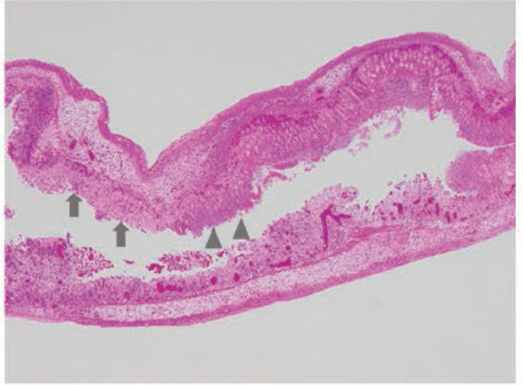

Vehicle control

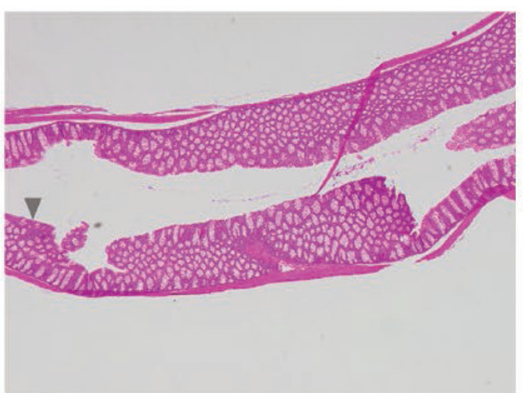

$3 \times 10^{10}$ three mixed $\mathrm{EV}$

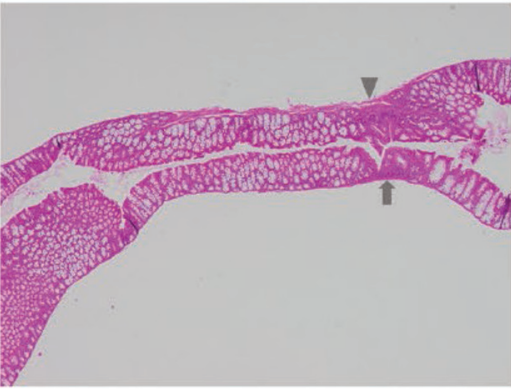

Prednisolone

B

Histopathological examination
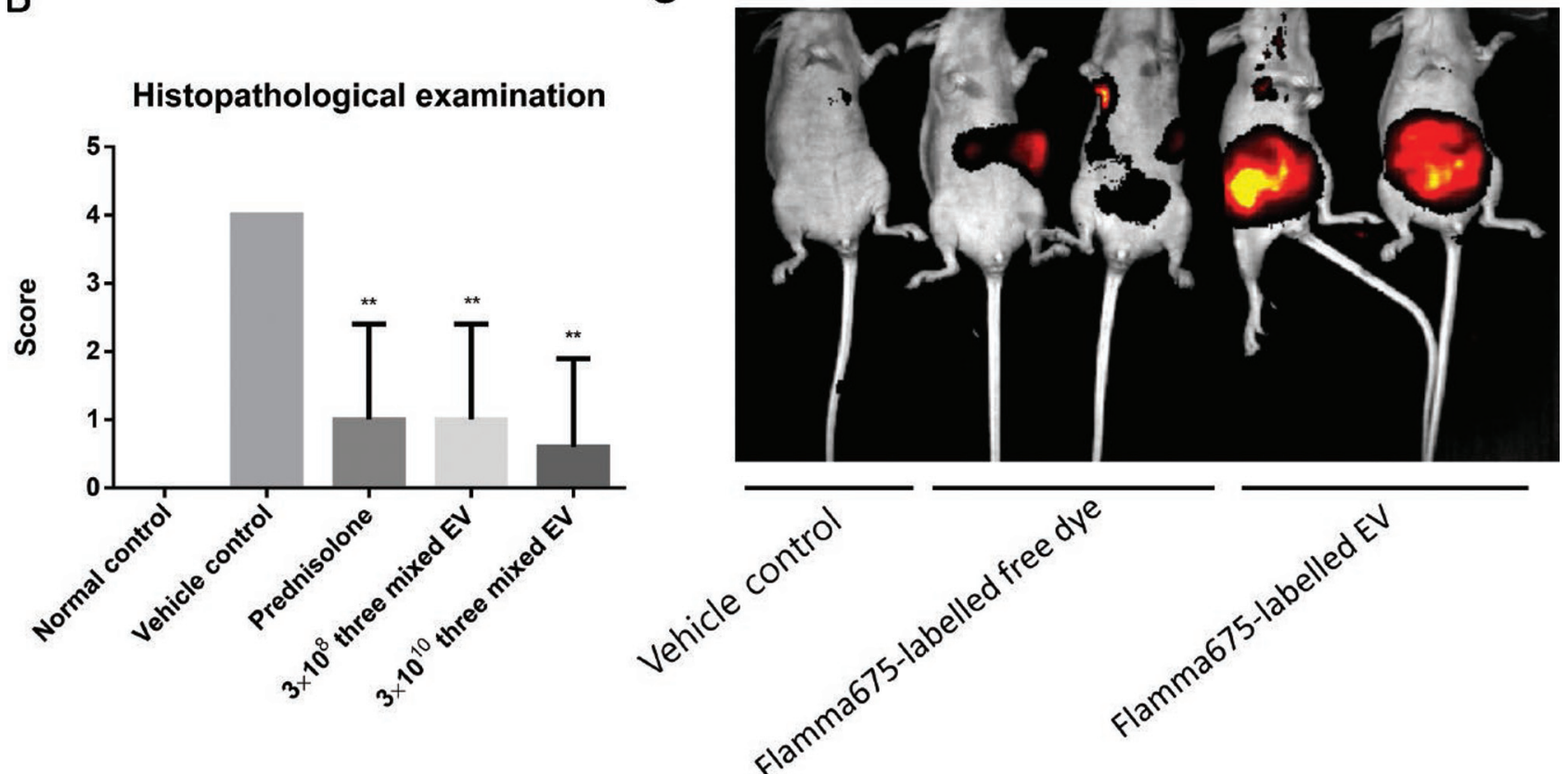

Figure 4. Histological examination of colon tissues. At d 10 of extracellular vesicle (EV) treatment, mice were killed for histological analysis. (A) The morphology of the large intestine revealed that EV-treated animals showed alleviated inflammatory bowel disease pathology progression. Black arrows indicate goblet cells in intact tissue, and gray arrows are regions where goblet cell loss occurred. Gray triangles indicate inflammatory cell infiltration. (B) The score of histopathological examination of mice. Data are presented as the means \pm SD $(\mathrm{n}=5)$. Asterisks indicate a significant difference at the ${ }^{* *} P<0.01 /{ }^{*} P<0.05$ level compared with the vehicle control. Optical imaging of the fluorescently labeled mixture of Lactobacillus kefir, Lactobacillus kefiranofaciens, and Lactobacillus kefirgranum EV (A mix ratios: 1:1:1) noninvasively monitored in mice after oral administration. Localization of the 3 mixed EV was evaluated in vivo using IVIS (Perkin Elmer, Waltham, MA) at 24 h after administrations of a single dose of the fluorescently labeled 3 mixed EV $(\mathrm{C})$. Vehicle control $(\mathrm{n}=1)$, Flamma675-labeled free dye $(\mathrm{n}=2)$, and Flamma675-labeled EV $(\mathrm{n}=2)$ for fluorescence imaging. Color version available online. 


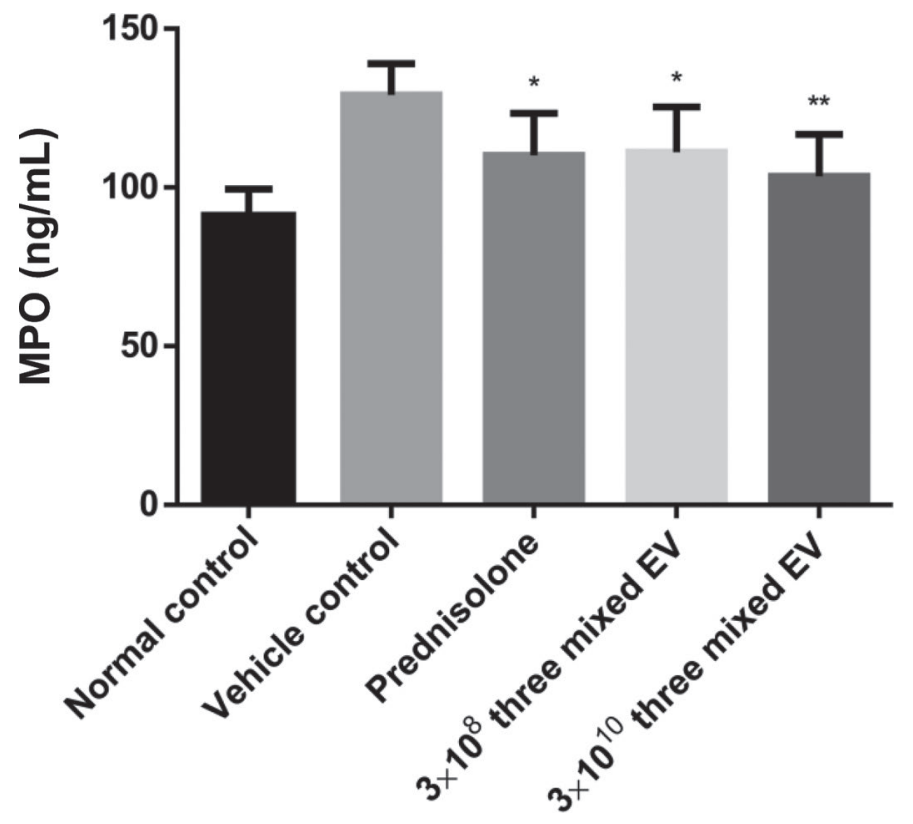

Figure 5. The kefir-derived extracellular vesicles reduce myeloperoxidase (MPO) activity. The MPO activity was measured at d 10 in the normal animals and those with experimentally induced inflammatory bowel disease, treated with normal saline (vehicle), prednisolone (positive), and the mixture of Lactobacillus kefir, Lactobacillus kefiranofaciens, and Lactobacillus kefirgranum EV in PBS (low and high dose), respectively. The activity of MPO was significantly decreased in the mice treated with the 3 mixed EV. Asterisks indicate a significant difference at the ${ }^{* *} P<0.01 /{ }^{*} P<0.05$ level compared with the vehicle control. Data are presented as the means $\pm \mathrm{SD}(\mathrm{n}=5)$.

show that the main size of kefir-derived Lactobacillus $\mathrm{EV}$ is around $100 \mathrm{~nm}$, with a range from 80 to $400 \mathrm{~nm}$ in diameter, which is consistent with previous results.

Monocyte chemoattractant protein-1 and IL-8 are known as the major factors that stimulate the inflammatory pathogenesis of IBD (Grimm et al., 1996). Especially IL- 8 tends to be produced from the intestinal mucous membrane of patients with IBD, and a correlation has been reported between IL-8 expression and inflammation level (Mazzucchelli et al., 1994; Daig et al., 1996). Furthermore, when antibody against IL-8 or its suppressor was administered in the animal experimental model, the symptoms were alleviated (Grimm et al., 1996). From these previous findings, our results suggest that kefir-derived Lactobacillus EV inhibited IL-8 expression in large intestine cell, and that they would be effective against IBD.

Probiotic lactobacillus is known to help intestinal regulation and antidiarrheal effect by preventing diarrhea and dehydration, and also by reducing intestinal pain and irritation (Saavedra et al., 2004; Thibault et al., 2004). Several studies have shown that gram-positive bacterial EV have functions such as bacteria-host interactions including protection against virulent strain infection, and immune responses against vancomycinresistant enterococci (Brown et al., 2015; Li et al., 2017). Therefore, those reports support our finding that the mixture of L. kefir, L. kefiranofaciens, and $L$. kefirgranum $\mathrm{EV}$ has an effect on the alleviation of IBD.

Oxidative stress signaling is associated with mobilization of inflammatory cells through inflammatory cytokines in inflammatory intestinal diseases. Similarly, the activation of inflammatory cells causes oxidative stress by increasing reactive oxygen species, which leads to intestinal damage (Fournier and Parkos, 2012; Bhattacharyya et al., 2014). Oxidative stress and lipid peroxidation that results from oxidative damage worsen the free radical chain reaction and activate the release of inflammatory cells such as neutrophils, which would subsequently increase MPO level (Bhattacharyya et al., 2014). Therefore, MPO activation has been widely used to evaluate anti-inflammatory drugs for IBD and its disease status. In the present study, we demonstrated that the administration of the mixture of L. kefir, $L$. kefiranofaciens, and L. kefirgranum EV significantly reduced the activity of MPO. These data suggest that the 3 mixed EV may inhibit the activity of MPO through regulating oxidative stress in intestinal epithelial cells.

Our results showed the EV derived from gram-positive functional bacteria have the potential to attenuate inflammation in intestinal epithelial cells. We speculate that developing protocols that can refine the process (i.e., treating cells with exogenous factors or environmental changes) is needed to make this biomaterial more commercially useful.

\section{CONCLUSIONS}

In this study, we demonstrated that EV produced from kefir can ease the TNF-induced inflammation in intestinal cells by inhibiting inflammatory cytokine production. We suggest that such an effect occurs via the NF-kB signaling pathway. In addition, using the IBD animal experimental model, we showed that the mixture of L. kefir, L. kefiranofaciens, and L. kefirgranum EV was effective in preventing enterorrhagia and diarrhea, and reducing MPO activity. Therefore, it is likely that EV produced from kefir-derived strains can be used to develop functional foods and materials, if the mechanism of their effects in IBD can be fully verified.

\section{ACKNOWLEDGMENTS}

This work was supported by Korea Institute of Planning and Evaluation for Technology in Food, Agriculture, Forestry and Fisheries (IPET) through High Value-Added Food Technology Development Program, 
funded by Ministry of Agriculture, Food and Rural Affairs (MAFRA, Gyeonggi-do, Korea; 115028-1).

\section{REFERENCES}

Anselmo, R. J., S. S. Viora, P. A. Ojeda, and L. I. Lausada. 2010. Efecto antagónico del kefir sobre endosporas y células vegetativas de Bacillus cereus y Clostridium perfringens. Inf. Tecnol. 21:131-138.

Bhattacharyya, A., R. Chattopadhyay, S. Mitra, and S. E. Crowe. 2014. Oxidative stress: An essential factor in the pathogenesis of gastrointestinal mucosal diseases. Physiol. Rev. 94:329-354.

Brown, L., J. M. Wolf, R. Prados-Rosales, and A. Casadevall. 2015. Through the wall: Extracellular vesicles in gram-positive bacteria, mycobacteria and fungi. Nat. Rev. Microbiol. 13:620-630.

Daig, R., T. Andus, E. Aschenbrenner, W. Falk, J. Scholmerich, and V. Gross. 1996. Increased interleukin 8 expression in the colon mucosa of patients with inflammatory bowel disease. Gut 38:216-222.

Deatherage, B. L., and B. T. Cookson. 2012. Membrane vesicle release in bacteria, eukaryotes, and archaea: A conserved yet underappreciated aspect of microbial life. Infect. Immun. 80:1948-1957.

Diniz, R., L. Garla, J. Schneedorf, and J. Carvalho. 2003. Study of anti-inflammatory activity of Tibetan mushroom, a symbiotic culture of bacteria and fungi encapsulated into a polysaccharide matrix. Pharmacol. Res. 47:49-52.

Farrell, R. J., and J. T. LaMont. 2002. Microbial factors in inflammatory bowel disease. Gastroenterol. Clin. North Am. 31:41-62.

Fontán, M. C. G., S. Martínez, I. Franco, and J. Carballo. 2006. Microbiological and chemical changes during the manufacture of Kefir made from cows' milk, using a commercial starter culture. Int. Dairy J. 16:762-767.

Fournier, B. M., and C. A. Parkos. 2012. The role of neutrophils during intestinal inflammation. Mucosal Immunol. 5:354-366.

Gao, J., F. Gu, H. Ruan, Q. Chen, J. He, and G. He. 2013. Induction of apoptosis of gastric cancer cells SGC7901 in vitro by a cell-free fraction of Tibetan kefir. Int. Dairy J. 30:14-18.

Grimm, M. C., S. K. Elsbury, P. Pavli, and W. F. Doe. 1996. Interleukin 8: Cells of origin in inflammatory bowel disease. Gut 38:90-98.

Gurung, M., D. C. Moon, C. W. Choi, J. H. Lee, Y. C. Bae, J. Kim, Y. C. Lee, S. Y. Seol, D. T. Cho, S. I. Kim, and J. C. Lee. 2011. Staphylococcus aureus produces membrane-derived vesicles that induce host cell death. PLoS One 6:e27958.

Guzel-Seydim, Z. B., T. Kok-Tas, A. K. Greene, and A. C. Seydim. 2011. Review: functional properties of kefir. Crit. Rev. Food Sci. Nutr. 51:261-268.

Hong, W.-S., H.-C. Chen, Y.-P. Chen, and M.-J. Chen. 2009. Effects of kefir supernatant and lactic acid bacteria isolated from kefir grain on cytokine production by macrophage. Int. Dairy J. 19:244-251.

Hong, W. S., Y. P. Chen, and M. J. Chen. 2010. The antiallergic effect of kefir Lactobacilli. J. Food Sci. 75:H244-H253.

Kim, O. Y., B. S. Hong, K. S. Park, Y. J. Yoon, S. J. Choi, W. H. Lee, T. Y. Roh, J. Lotvall, Y. K. Kim, and Y. S. Gho. 2013. Immunization with Escherichia coli outer membrane vesicles protects bacteria-induced lethality via Th1 and Th17 cell responses. J. Immunol. 190:4092-4102.

Kwon, C.-S., M.-Y. Park, J.-S. Cho, S.-T. Choil, and D. S. Chang. 2003. Identification of effective microorganisms from kefir fermented milk. Food Sci. Biotechnol. 12:476-479.

Kwon, Y.-I., E. Apostolidis, and K. Shetty. 2006. Anti-diabetes functionality of kefir culture-mediated fermented soymilk supplemented with rhodiola extracts. Food Biotechnol. 20:13-29.

Lee, E. Y., D. S. Choi, K. P. Kim, and Y. S. Gho. 2008. Proteomics in gram-negative bacterial outer membrane vesicles. Mass Spectrom. Rev. 27:535-555.

Lee, E. Y., D. Y. Choi, D. K. Kim, J. W. Kim, J. O. Park, S. Kim, S. H. Kim, D. M. Desiderio, Y. K. Kim, K. P. Kim, and Y. S. Gho.
2009. Gram-positive bacteria produce membrane vesicles: Proteomics-based characterization of Staphylococcus aureus-derived membrane vesicles. Proteomics 9:5425-5436.

Li, M., K. Lee, M. Hsu, G. Nau, E. Mylonakis, and B. Ramratnam. 2017. Lactobacillus-derived extracellular vesicles enhance host immune responses against vancomycin-resistant enterococci. BMC Microbiol. 17:66.

Mashburn-Warren, L. M., and M. Whiteley. 2006. Special delivery: Vesicle trafficking in prokaryotes. Mol. Microbiol. 61:839-846.

Mazzucchelli, L., C. Hauser, K. Zgraggen, H. Wagner, M. Hess, J. A. Laissue, and C. Mueller. 1994. Expression of interleukin-8 gene in inflammatory bowel disease is related to the histological grade of active inflammation. Am. J. Pathol. 144:997-1007.

McCarthy, J., L. O'Mahony, L. O'Callaghan, B. Sheil, E. Vaughan, N. Fitzsimons, J. Fitzgibbon, G. O'Sullivan, B. Kiely, and J. Collins. 2003. Double blind, placebo controlled trial of two probiotic strains in interleukin 10 knockout mice and mechanistic link with cytokine balance. Gut 52:975-980.

Oliveira, D. L., C. G. Freire-de-Lima, J. D. Nosanchuk, A. Casadevall, M. L. Rodrigues, and L. Nimrichter. 2010. Extracellular vesicles from Cryptococcus neoformans modulate macrophage functions. Infect. Immun. 78:1601-1609.

Pogačić, T., S. Šinko, Š. Zamberlin, and D. Samaržija. 2013. Microbiota of kefir grains. Mljekarstvo 63:3-14.

Rivera, J., R. J. Cordero, A. S. Nakouzi, S. Frases, A. Nicola, and A. Casadevall. 2010. Bacillus anthracis produces membrane-derived vesicles containing biologically active toxins. Proc. Natl. Acad. Sci. USA 107:19002-19007.

Saavedra, J. M., A. Abi-Hanna, N. Moore, and R. H. Yolken. 2004. Long-term consumption of infant formulas containing live probiotic bacteria: Tolerance and safety. Am. J. Clin. Nutr. 79:261-267.

Sartor, R. B. 2004. Therapeutic manipulation of the enteric microflora in inflammatory bowel diseases: Antibiotics, probiotics, and prebiotics. Gastroenterology 126:1620-1633.

Schwechheimer, C., C. J. Sullivan, and M. J. Kuehn. 2013. Envelope control of outer membrane vesicle production in Gram-negative bacteria. Biochemistry 52:3031-3040.

Senol, A., M. Isler, R. Sutcu, M. Akin, E. Cakir, B. M. Ceyhan, and M. C. Kockar. 2015. Kefir treatment ameliorates dextran sulfate sodium-induced colitis in rats. World J. Gastroenterol. 21:1302013029.

Serafini, F., F. Turroni, P. Ruas-Madiedo, G. A. Lugli, C. Milani, S. Duranti, N. Zamboni, F. Bottacini, D. van Sinderen, and A. Margolles. 2014. Kefir fermented milk and kefiran promote growth of Bifidobacterium bifidum PRL2010 and modulate its gene expression. Int. J. Food Microbiol. 178:50-59.

Taș, T. K., F. Y. Ekinci, and Z. B. Guzel-Seydim. 2012. Identification of microbial flora in kefir grains produced in Turkey using PCR. Int. J. Dairy Technol. 65:126-131.

Thibault, H., C. Aubert-Jacquin, and O. Goulet. 2004. Effects of long-term consumption of a fermented infant formula (with $B i$ fidobacterium breve c50 and Streptococcus thermophilus 065) on acute diarrhea in healthy infants. J. Pediatr. Gastroenterol. Nutr. $39: 147-152$

Wyder, M.-T., L. Meile, and M. Teuber. 1999. Description of Saccharomyces turicensis sp. nov., a new species from kefyr. Syst. Appl. Microbiol. 22:420-425.

Yang, X.-J., M.-T. Fan, J.-L. Shi, and B. Dang. 2007. Isolation and identification of preponderant flora in Tibetan fermented linggu milk. China Brewing 6:10-16.

Zhou, J., X. Liu, H. Jiang, and M. Dong. 2009. Analysis of the microflora in Tibetan kefir grains using denaturing gradient gel electrophoresis. Food Microbiol. 26:770-775.

Zhou, L., R. Srisatjaluk, D. E. Justus, and R. J. Doyle. 1998. On the origin of membrane vesicles in gram-negative bacteria. FEMS Microbiol. Lett. 163:223-228. 